\title{
On the energy spectrum of cosmic rays in the model of relativistic nonlocal diffusion
}

\author{
R. T. Sibatov* \\ Ulyanovsk State University, Ulyanovsk, Russia \\ E-mail: ren_sib@bk.ru \\ V. V. Uchaikin \\ Ulyanovsk State University, Ulyanovsk, Russia \\ E-mail: vuchaikin@gmail.com
}

\begin{abstract}
Commonly used cosmic ray transport equations originate in the standard diffusion model as the most known random walk process. However, at least two its features are incompatible with such fixed facts as relativistic boundedness of velocity and multiscale heterogeneity of the interstellar medium. Here is considered the nonlocal relativistic CR-trasport model which is free from both these imperfections, and involving energy dependence of the mean free path length.
\end{abstract}

The 34th International Cosmic Ray Conference,

30 July- 6 August, 2015

The Hague, The Netherlands

${ }^{*}$ Speaker. 


\section{Introduction}

As is known, there exist several specified explanations for the origin of the knee in the CR spectrum [1], but none is universally recognized. In recent paper [2], authors distinguish three of them. The hypothesis that suppression of the CR flux in the region above the knee is caused by changes in particle interactions is now excluded by the LHC data. Another one connects the knee with the maximum rigidity to which CRs can be accelerated in the dominant population of Galactic CR sources (probably, in a single nearby source such as Monogem Ring) [2]. The third explanation emphasized in [2] implies a change of the diffusion properties of charged CRs [3]. The knee energy can be related to the rigidity at which the CR Larmor radius $r_{L}$ is equal to the coherence length $l_{c}$ of the turbulent Galactic magnetic field. This leads to different energy dependence of the diffusion coefficient below and above $E_{\text {knee }}$. Confinement time changes, which results in a steepening of the spectrum. It is worth to note, that almost all of the approaches are based on the standard diffusion model or their minimal modifications. Nevertheless, authors $[4,5]$ see the necessity of a more deep modification of the CR transport theory. The authors of [4] write: "Diffusion equations are intrinsically non-relativistic and superluminal velocities appear naturally there. The cardinal solution of this problem - the relativistic generalization of the diffusion equation - still expects to be found after more than 70 years of unsuccessful attempts."

Commonly used cosmic ray transport equations originate in the standard diffusion model, the most known random walk process. There exist at least two reasons to be unsatisfied by cosmic ray transport equations originated from the ordinary diffusion model (ditto Brownian motion). Solution of the equation describes the spatial-temporal distribution of Brownian particle, but does not provide an explicit feature of the motion. First of them is in the conflict with the special relativity via admitting velocities to exceed the speed of light: immediately after the source flash, its products turn out to be distributed over all space [6]. Second, the Brownian motion is a homogeneous (in time) process with independent increments, that means the absence of any correlations between properties of adjacent segments of the medium, as if it was an ideal gas. But it is not the case: different elements of the interstellar medium could be linked via long magnetic field lines having in addition the turbulent (one might say, fractal) character. Involving the free motion velocity restriction, a more accurate description of CR's propagation may be achieved by using the telegraph equation [7, 8]. In [8], authors analyze the one-dimensional case, and it is known that the telegraph equation faces the problems in description of three-dimensional finite-velocity diffusion. The turbulent heterogeneous character of the medium can be taken into account by passage to inverse power type distribution for free paths $[10,11,5]$. Finally, combining these two peculiarities has led to the fractional-order differential equation with the material derivative operator being of primary importance. Due to the fractional-order operator belongs to the nonlocal family, we call this process the relativistic nonlocal diffusion (NRD).

Paper is organized in the following way. In next section, we consider CR propagators of different diffusion models and discuss the importance of the relativistic restriction at energies near the knee of the spectrum. Then, we estimate the equilibrium spectrum taking the mentioned effects into account. We will show that the role of relativistic restriction increases in the anomalous diffusion models and provide preliminary estimates. 


\section{Propagators: from ordinary to relativistic nonlocal diffusion}

Let us list the propagator models, which we compare in estimations of equilibrium spectra in this work

\section{- The Gaussian density function}

$$
G_{\mathrm{G}}\left(\mathbf{r}, t, E ; \mathbf{r}_{0}, t_{0}, E_{0}\right)=\frac{\delta\left(E-E_{0}\right)}{[4 \pi D(E) \tau]^{3 / 2}} \exp \left[-\frac{\left(\mathbf{r}-\mathbf{r}_{0}\right)^{2}}{4 D(E) \tau}\right] ; \quad \tau=t-t_{0},
$$

is the fundamental solution of the ordinary diffusion equation. The model doesn't take into account neither the relativistic boundedness of speed nor fractal properties of ISM. The latter is assumed to be uniformly homogeneous without any signs of turbulence, so the diffusion is a small-scale process. First is applied to CR transport in 60th years of the former century.

- The Dunkel-Hänggi-Jüttner (DHJ) propagator [12,13]

$$
\begin{gathered}
G_{\mathrm{J}}\left(\mathbf{r}, t, E ; \mathbf{r}_{0}, t_{0}, E_{0}\right)=\frac{N_{k}(E) 1(c \tau-r) c^{2} \tau}{8 D(E) \pi(c \tau)^{3} K_{1}\left(\frac{c^{2} \tau}{2 D(E)}\right)\left[1-\frac{r^{2}}{(c \tau)^{2}}\right]^{2}} \exp \left(-\frac{c^{2} \tau}{2 D(E) \sqrt{1-\frac{r^{2}}{(c \tau)^{2}}}}\right) \\
\tau=t-t_{0}, r=\left|\mathbf{r}-\mathbf{r}_{0}\right|
\end{gathered}
$$

includes the relativistic boundedness and describes both regimes: small-scale diffusive motion and ballistic motion as particular extreme cases. Its first application to CR-transport in the Galaxy was undertaken in [4]. However, this propagator does not account for multiscale heterogeneity (fractality) of ISM.

- The Lévy-Feldheim trivariate isotropic distributions $g_{3}(r, \alpha), \alpha \in(0,2]$ :

$$
G_{\mathrm{LF}}\left(\mathbf{r}, t, E ; \mathbf{r}_{0}, t_{0}, E_{0}\right)=\delta\left(E-E_{0}\right)\left[K(E)\left(t-t_{0}\right)\right]^{-3 / \alpha} g_{3}\left(\left|\mathbf{r}-\mathbf{r}_{0}\right|\left[K(E)\left(t-t_{0}\right)\right]^{-1 / \alpha}\right), t>t_{0},
$$

are the Green functions of the diffusion equation with the fractional Laplacian, describing superdiffusive transport of CRs [9]. It is important to note, that when passing from the normal value $\alpha=2$ to anomalous ones $\alpha<2$ it is not only the form what is changed (power tails appears and increases) but the extension rate of diffusion packet also changes (increases): its width grows with time $\tau=\left|t-t_{0}\right|$ accordingly to the law $\tau^{1 / \alpha}, \alpha<2$, while in the normal case the packet's width is proportional to $\tau^{1 / 2}$. These propagators introduced into CR-transport in the Galaxy in $[10,11]$ to solve the energy spectrum problem, taking into account the ISM heterogeneities were testified to take large-scaled (fractal) character (see [14]). The supernova remnants analysis shows the presence in this region of gas components with different physical parameters $\left(T_{e} \sim 5 \div 10^{6} \mathrm{~K}\right.$, $n_{e} \sim 0.1 \div 10^{3} \mathrm{~m}^{-3}$ ), what can be the sequence of turbulent heterogeneity of ISM. These facts and other data concerning the heterogeneities of matter density $\rho$ and magnetic field intensity $H \propto \rho^{q}$, $q \sim 1 / 3 \div 1 / 2$ ([15]) within the length range 100-150 pc, giving rise to uncertainties of diffusion model to be applicable to cosmic rays transfer description, stimulated the use of the superdiffusion model, based on the fractional Laplacian operator [11]. However, this model assumes instantaneous jumps interrupted by confining in magnetic traps.

In a single-nearest source approximation, this model turned out to be able to explain the experimentally observed 'knee' in the energy spectrum, i. e. increasing of the exponent $\eta$ in power 
representation of the $E^{-\eta}$ spectrum while passing from the region $\sim 10^{2} \mathrm{Gev} /$ nucleon into that of $\sim 10^{5} \mathrm{Gev} /$ nucleon. The explanation is connected with the presence of the anomalously large free paths of particles (Lévy "flights") in the regions of reduced magnetic fields.

- Nonlocal relativistic diffusion (NRD) propagator accounting for both these effects (finite velocity and fractality of ISM) was proposed in [5] and developed in our papers (see, e.g. [16]). It is based on integral equations of the form

$$
\begin{gathered}
G_{\mathrm{NR}}(\mathbf{r}, t, E)=\int \frac{d \mathbf{r}^{\prime}}{v} P\left(\mathbf{r}^{\prime}, E\right) f\left(\mathbf{r}-\mathbf{r}^{\prime}, t-\frac{r^{\prime}}{v}, E\right), \\
f(\mathbf{r}, t, E)=\int f\left(\mathbf{r}-\mathbf{r}^{\prime}, t-\frac{r^{\prime}}{v}, E\right) p\left(\mathbf{r}^{\prime}, E\right) d \mathbf{r}^{\prime}+\delta\left(\mathbf{r}^{\prime}\right) \boldsymbol{\delta}(t),
\end{gathered}
$$

where $f(\mathbf{r}, t, E)$ is the density of collisions per a unit volume and a unit time, $p\left(\mathbf{r}^{\prime}, E\right)$ and $P\left(\mathbf{r}^{\prime}, E\right)$ are density function and survival probability for free path lengths. Under appropriate choice of transtion probability $P$, this model, including an integral (i.e. nonlocal) operator is able to describe relativistic kinetics in a turbulent media.

\section{Some results of numerical calculations}

Here, we evaluative the background spectrum expected from CR sources distributed in the Galactic disc using propagators listed in the previous section. Source spectrum is usually modeled by a power law with exponential cutoff [17]. We consider a pure power law for injected particles.

Diffusion coefficient, "determined from statistically reliable data (up to $100 \mathrm{GeV} /$ nuclon) on secondary nuclei" [18], depends on energy according to the power law $\propto R^{\delta}$, where $R=p c / Z$ is a magnetic rigidity, $p$ momentum of a particle, $Z$ nucleus charge, $\beta=v / c, v$ particle velocity. Exponent $\delta=0.6$ in pure diffusion model, and $\delta=0.34$ accounting for diffusive reacceleration of particles by random magnetohydrodynamic waves in interstellar medium and Alfven velocity $V_{a} \approx 36 \mathrm{~km} \cdot \mathrm{s}^{-1}$ [18]. Popular dependencies for diffusion coefficients in different models for $R>$ $R_{0}=3 \mathrm{GV}$ are listed in Table 1 .

Table 1: Diffusion coefficients in different models for $R>R_{0}=3 \mathrm{GV}$.

\begin{tabular}{|l|c|c|}
\hline \multicolumn{1}{|c|}{ Model } & Diffusion Coefficient $D\left[\mathrm{~cm}^{2} / \mathrm{s}\right]$ & $\dot{\lambda}_{10^{6} \mathrm{GV}}[\mathrm{pc}]$ \\
\hline Ptuskin et al. (plain diffusion) [19] & $2.2 \cdot 10^{28} \beta^{-2}\left(R / R_{0}\right)^{0.6}$ & 1468 \\
\hline Ptuskin et al. (diffusive reacceleration) [19] & $5.2 \cdot 10^{28} \beta^{-2}\left(R / R_{0}\right)^{0.34}$ & 127 \\
\hline Ptuskin et al. (diffusive reacceleration with damping) [19] & $2.9 \cdot 10^{28} \beta^{-2}\left(R / R_{0}\right)^{0.5}$ & 542 \\
\hline Blasi and Amato, $\delta=0.6[17]$ & $0.55 \cdot 10^{28} H_{\mathrm{kpc}}\left(R / R_{0}\right)^{0.6}$ & 1101 \\
\hline Blasi and Amato, $\delta=1 / 3[17]$ & $1.33 \cdot 10^{28} H_{\mathrm{kpc}}\left(R / R_{0}\right)^{1 / 3}$ & 90 \\
\hline
\end{tabular}

Relativistic restriction plays a crucial role when the size of propagation area of interest is comparable with a mean free path length $\lambda$. The latter may be estimated as a function of energy $\lambda(E)$ from the acceptable values of the diffusion coefficient used, for example, in GALPROP [19] or in some other calculations, e.g. presented in [17]. The lower-bound estimate for isotropic threedimensional diffusion can be found via formula $\breve{\lambda}(E)=3 D(E) / c$, where $c$ is the speed of light. 

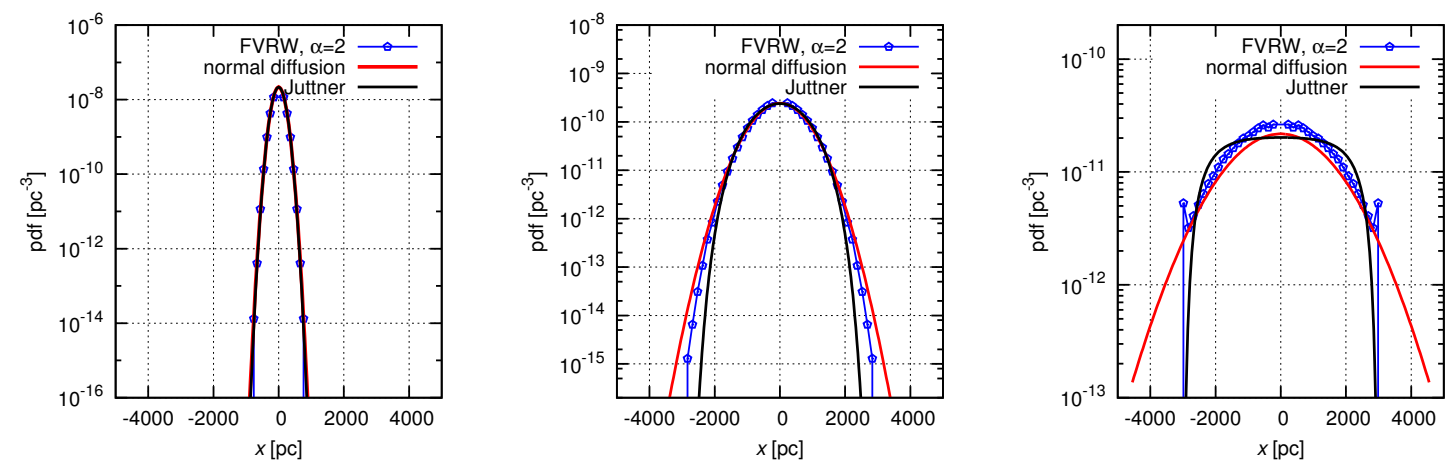

Figure 1: Comparison of evolutions of the DHJ distribution with the Monte Carlo solutions of the constantvelocity random walk for the case of exponential distribution of path lengths. Results for three values of rigidity are presented $R=1 \cdot 10^{4} \mathrm{GV}$ (left panel), $R=5 \cdot 10^{4} \mathrm{GV}$ (middle panel) $R=3 \cdot 10^{5} \mathrm{GV}$ (right panel); $\delta=0.6$ (plain diffusion). The age is $50000 \mathrm{yr}$.
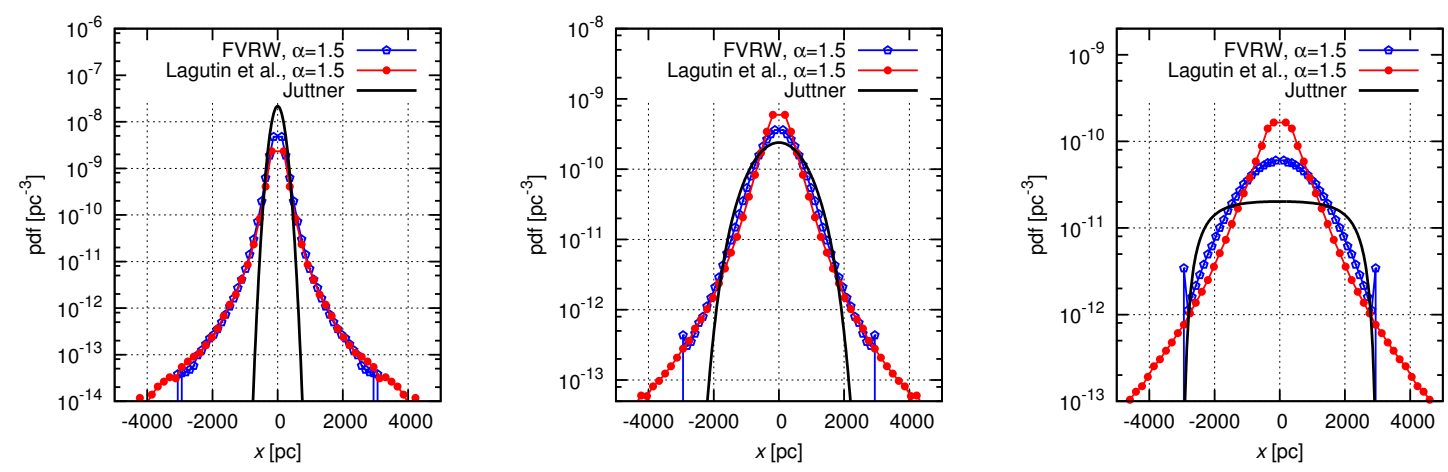

Figure 2: Comparison of evolutions of the DHJ distribution with the Monte Carlo solutions of the constantvelocity random walk for the case of power law distribution $(\alpha=1.5)$ of path lengths. Results for three values of rigidity are presented $R=1 \cdot 10^{4} \mathrm{GV}$ (left panel), $R=5 \cdot 10^{4} \mathrm{GV}$ (middle panel) $R=3 \cdot 10^{5} \mathrm{GV}$ (right panel); $\delta=0.6$ (plain anomalous diffusion). The age is $50000 \mathrm{yr}$.

Calculations show that the mean free path at rigidity $10^{6} \mathrm{GV}$ may be comparable with the size of the Galactic halo ( $H=4 \mathrm{kpc}$ ) in some of the mentioned models (see table). This indicate that relativistic boundedness of propagators may play role in formation of the spectrum near the 'knee'. Comparison of the listed propagators is presented in Figs. 1 and 2.

Evaluative calculations of the spectrum expected from CR sources distributed in the Galactic disc is performed in the following way. The disc is considered as infinitely thin and the sources are uniformly distributed in this disk with radius $R_{d}$. The flux of CRs propagating in the space surrounding the disk is calculated at the center according to formula

$$
n_{\mathrm{CR}}=\int_{0}^{R_{\mathrm{d}}} d r \int_{t_{\text {in }}}^{\infty} d \tau \frac{2 \pi r}{\pi R_{\mathrm{d}}^{2}} N(E) \mathscr{R} G(\mathbf{r}, t, E),
$$

where $t_{\text {in }}=0$ is taken by Blasi and Amato [17]. Here, $\mathscr{R}$ is a rate of SN explosions. We estimate the spectrum of protons (no spallation). We do not account for the Galactic halo boundaries in these evaluating calculations. The Gaussian and Lévy-Feldheim propagators admit nonzero values 
beyond the relativistic restriction. In these cases, the lower limit $t_{\text {in }}=0$ can lead to incorrect results. Limit $t_{\text {in }}=r / v$ implying the ballistic restriction of propagators is more correct.

Here, we imply the standard assumptions about the origin of galactic cosmic rays [20]. First, CRs are injected from Galactic sources, such as supernova remnants (SNRs) with power law spectra, $N(E) \sim E^{-\gamma}$. The second point, cosmic rays propagate diffusively throughout the Galaxy with a diffusion coefficient $D(E) \propto E^{\delta}$. The simple combination of these propositions leads to the equilibrium spectrum $n(E) \propto N(E) / D(E) \propto E^{-\gamma+\delta}$ reflecting the balance between injection and escape of CRs from the confinement volume (e.g. the galactic halo) [20]. Such estimation is modified in the NRD model.

Results of calculations for proton spectra for various propagators are presented in Fig. 3. We considered Gaussian, DHJ, renormalized and truncated Gaussian and Lévy-Feldheim propagators approximating solutions of nonlocal relativistic diffusion equation. Remarkable result is that accounting for relativistic restriction steepens the spectrum at high energies. Under assumption that CRs are injected from sources with pure power law spectra, $N(E) \sim E^{-\gamma}$, the position of the spectrum break, its sharpness and slopes of asymptotics depend on diffusion parameters $D_{\alpha}(E)$ and $\alpha$ (see Fig. 3, right panel).
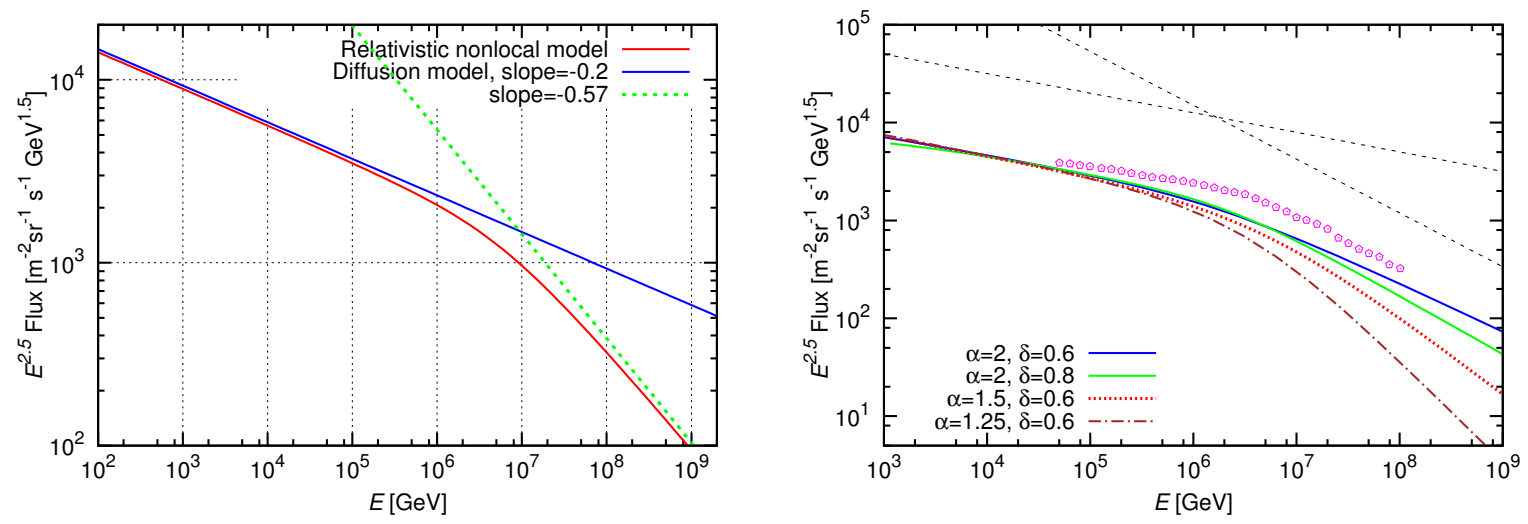

Figure 3: Estimates of equilibrium spectra. Left panel: spectra calculated for the case of $D(E) \propto E^{\delta}$ with $\delta=0.75$, and $N(E) \propto E^{-\gamma}$ with $\gamma=1.95$ for the Gaussian nontruncated propagator (blue line) and the relativistic one (red line). Right panel: spectra for truncated Gaussian and Lévy-Feldheim propagators $(\alpha=1.5,1.25)$ approximating solutions of nonlocal relativistic diffusion equation $(v=c / 3)$.

So, the NRD model provides an alternative cause of the spectrum steepening at high energies. Sure, the determination of position of the break provided by relativistic effect, its role in formation of the knee observed in experiment requires additional detailed calculations and matching. But obvious, that cause of this effect is universal and must be kept in mind in all calculations of propagation of high energy CRs. Explanation of the spectrum steepening at high energies in the NRD model is schematically represented in Fig. 4. The grey field corresponds to the space-time area containing CR sources which do not take part in the formation of the spectrum due to the relativistic restriction. Their accounting in the ordinary diffusion model leads to a pure power law spectrum $\propto E^{-\gamma-\delta}$ under the assumption about the pure power law spectrum of injected particles. Taking the relativistic restriction into account in the NRD model excludes these sources from the consideration, but this exclusion affects predominantly on high energy part of CRs due to $D(E) \propto E^{\delta}$. 
Number of these sources is not small. The lower-bound estimate for $R_{d}=15 \mathrm{kpc}, \mathscr{R}=1 / 100 \mathrm{yr}^{-1}$, is about 300 young sources (with ages $\lesssim 45000 \mathrm{yr}$ ). Mentioned truncation procedure also implies that we neglects by sources located at the boundary of the relativistic cone, i.e. there are no CR fronts from quite young sources penetrating the Sun system at the moment.

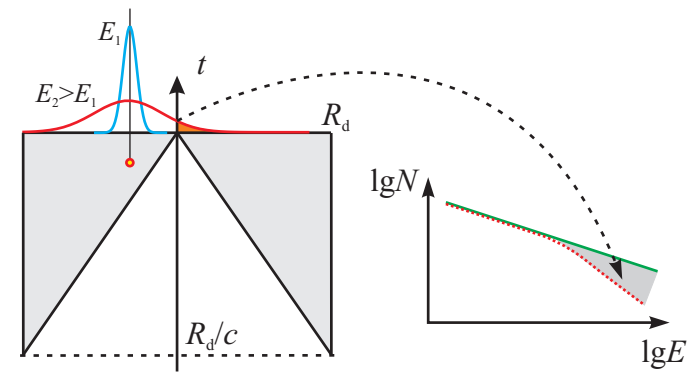

Figure 4: Sketch providing a possible explanation of steepening in the spectrum at high energies in the NRD model.

\section{Conclusion}

The NRD model accounts for constant velocity of particle motion, nonlocal character of particle transport caused by the turbulent nature of the interstellar magnetic field, energy dependence of the mean free path length. First calculations in frame of this model demonstrates the following.

1. The DHJ propagator is based on the Gibbs statistics derived for Gaussian random fields with short correlations. For this reason it differs from ordinary diffusion model only at short times.

2. Contribution of nearest sources into the 'knee' formation doesn't practically depend on the relativistic boundedness of speed but essentially determined by fractal property of ISM, as it was obtained in [10].

3. On the contrary, the averaged influence of distant sources depends rather on the speed boundedness than on turbulent character of ISM. Moreover, this fact brings some contribution into the 'knee' formation as well. The remarkable result is that under conventional assumptions about the origin of galactic cosmic rays the relativistic principle of speed boundedness may explain the origin of 'knee' in the equilibrium spectrum of cosmic rays. Theoretical 'knee' energy depends on a propagator model and corresponding energy dependence of the diffusion coefficient. Calculations show that the ordinary diffusion model with commonly used parameters leads to $E_{\text {knee }}$ values (produced by relativistic restriction) a little greater than the observed ones. Introducing the relativistic nonlocal diffusion model accounting for fractal character of interstellar medium diminishes this value to lower energies.

Acknowledgment. We thank the Russian Foundation for Basic Research (13-01-00585) and the Ministry of Education and Science of the Russian Federation (2014/296) for financial support.

\section{References}

[1] J.R. Hörandel, Models of the knee in the energy spectrum of cosmic rays.Astroparticle Physics, 21(3) (2004) 241-265. 
[2] G. Giacinti, M. Kachelriess, D.V. Semikoz, G. Sigl, Cosmic ray anisotropy as signature for the transition from galactic to extragalactic cosmic rays. Journal of Cosmology and Astroparticle Physics, 2012(07) (2012) 031.

[3] J. Abraham, P. Abreu, M. Aglietta, E.J. Ahn, D. Allard, I. Allekotte, et al., Measurement of the depth of maximum of extensive air showers above $1018 \mathrm{eV}$. Physical Review Letters, 104(9) (2010) 091101.

[4] R. Aloisio, V. Berezinsky, A. Gazizov, The problem of superluminal diffusion of relativistic particles and its phenomenological solution. The Astrophysical Journal, 693(2) (2009) 1275.

[5] V.V. Uchaikin, On the fractional derivative model of the transport of cosmic rays in the Galaxy. JETP letters 91 (3) (2010) 105-109.

[6] J. Dunkel, P. Hänggi, Theory of relativistic Brownian motion: the (1+1)-dimensional case.Physical Review E, 71(1) (2005) 016124.

[7] S. Goldstein. On diffusion by discontinuous movements, and on the telegraph equation. The Quarterly Journal of Mechanics and Applied Mathematics, 4(2) (1951) 129-156.

[8] Y.E. Litvinenko, F. Effenberger, R. Schlickeiser, The telegraph approximation for focused cosmic-ray transport in the presence of boundaries. arXiv preprint (2015) arXiv:1505.05134.

[9] V. V. Uchaikin. Nonlocal Models of Cosmic Ray Transport in the Galaxy. Journal of Applied Mathematics and Physics, 3(02) (2015) 187.

[10] A. Lagutin, Y.A. Nikulin, V.V. Uchaikin, Nuclear Physics B-Proceedings Supplements 97(1) (2001) 267.

[11] A. Lagutin, V. Uchaikin, Anomalous diffusion equation: Application to cosmic ray transport. Nuclear Instruments and Methods in Physics Research Section B: Beam Interactions with Materials and Atoms 201(1) (2003) 212.

[12] F. Jüttner, Das Maxwellsche Gesetz der Geschwindigkeitsverteilung in der Relativtheorie, Ann. Phys. (Leipzig) 34 (1911) 856-882.

[13] J. Dunkel, P. Talkner, P. Hänggi, Relativistic diffusion processes and random walk models. Physical Review D 75(4) (2007) 043001.

[14] A. V. Kulakov, A. A. Rumjantsev. Fractals and energy spectrum of fast particles. Reports of the Russian Academy of Sciences 336 (1994) 183.

[15] A. A. Ruzmaikin, A. M. Shukurov, D. D. Sokolov, Magnetic Fields of Galaxies. Kluwer, Dordrecht (1988).

[16] V.V. Uchaikin, R.T. Sibatov, On fractional differential models for cosmic ray diffusion. Gravitation and Cosmology, 18(2) (2012) 122-126.

[17] P. Blasi, E. Amato, Diffusive propagation of cosmic rays from supernova remnants in the Galaxy. I: spectrum and chemical composition. Journal of Cosmology and Astroparticle Physics, 2012(01) (2012) 010.

[18] V.S. Ptuskin, On the origin of galactic cosmic rays.Physics-Uspekhi, 50(5) (2007) 534.

[19] V.S. Ptuskin, I.V. Moskalenko, F.C. Jones, A.W. Strong, V.N. Zirakashvili, Dissipation of magnetohydrodynamic waves on energetic particles: impact on interstellar turbulence and cosmic-ray transport. The Astrophysical Journal 642(2) (2006) 902.

[20] P. Blasi, Recent developments in cosmic ray physics. Nuclear Physics B-Proceedings Supplements 256 (2014) 36. 